\title{
Studying multisensory processing and its role in the representation of space through pathological and physiological crossmodal extinction
}

\author{
Stéphane Jacobs ${ }^{1,2,3 *}$, Claudio Brozzoli ${ }^{4}$, Fadila Hadj-Bouziane ${ }^{1,2,3}$, Martine Meunier ${ }^{1,2,3}$ and \\ Alessandro Farnè ${ }^{1,2,3 *}$ \\ ImpAct Team, INSERM U1028, Lyon Neuroscience Research Center, Lyon, France \\ 2 ImpAct Team, CNRS UMR5292, Lyon Neuroscience Research Center, Lyon, France \\ 3 University Lyon I, Lyon, France \\ ${ }^{4}$ Brain, Body and Self Laboratory, Department of Neuroscience, Karolinska Institutet, Stockholm, Sweden
}

\section{Edited by:}

Nadia Bolognini, University of

Milano-Bicocca, Italy

Angelo Maravita, University of

Milano-Bicocca, Italy

Reviewed by:

Francesca Frassinetti, University of Bologna, Italy

Elena Natale, University of Milano-

Bicocca, Italy

*Correspondence:

Stéphane Jacobs and Alessandro Farnè, INSERM U1028, CNRS

UMR5292, Centre de Recherche en Neurosciences de Lyon, Equipe IMPACT, 16 Avenue du Doyen Lépine, 69500 Bron, France.

email: stephane.jacobs@inserm.fr; alessandro.farne@inserm.fr
The study of crossmodal extinction has brought a considerable contribution to our understanding of how the integration of stimuli perceived in multiple sensory modalities is used by the nervous system to build coherent representations of the space that directly surrounds us. Indeed, by revealing interferences between stimuli in a disturbed system, extinction provides an invaluable opportunity to investigate the interactions that normally exist between those stimuli in an intact system. Here, we first review studies on pathological crossmodal extinction, from the original demonstration of its existence, to its role in the exploration of the multisensory neural representation of space and the current theoretical accounts proposed to explain the mechanisms involved in extinction and multisensory competition. Then, in the second part of this paper, we report recent findings showing that physiological multisensory competition phenomena resembling clinical crossmodal extinction exist in the healthy brain. We propose that the development of a physiological model of sensory competition is fundamental to deepen our understanding of the cerebral mechanisms of multisensory perception and integration. In addition, a similar approach to develop a model of physiological sensory competition in non-human primates should allow combining functional neuroimaging with more invasive techniques, such as transient focal lesions, in order to bridge the gap between works done in the two species and at different levels of analysis.

Keywords: extinction, multisensory, neuropsychology, perception, peripersonal space, non-human primate

\section{INTRODUCTION}

As in many fields of neuropsychology, the study of multisensory processing dysfunctions in brain-lesioned patients has been of invaluable help in better understanding the mechanisms and functional roles of multisensory perception and integration in human cognition. In particular, crossmodal extinction has constituted a very useful model for investigating how multiple representations of the space that surrounds us are built in our brains through the integration of sensory information perceived in different modalities (Làdavas and Farnè, 2004; Farnè et al., 2007).

Extinction is a neuropsychological syndrome closely related to spatial neglect ${ }^{1}$, both arising in a vast majority of cases following damage to the right hemisphere, most typically in the posterior parietal region (Becker and Karnath, 2007). While neglect patients fail to consciously perceive sensory events located in the contral-

\footnotetext{
${ }^{1}$ Although this is beyond the scope of this review, it should be noted that the question of whether extinction and neglect should be conceived of as common or separate deficits is still debated. Indeed, while extinction is often considered as a milder form or a residual manifestation of neglect after recovery (e.g., Heilman et al., 1993), dissociations have been reported (e.g., di Pellegrino and De Renzi, 1995; Cocchini et al., 1999; Geeraerts et al., 2005) and distinct neural substrates have been proposed (Vallar et al., 1994; Karnath et al., 2003), challenging the view that both syndromes are just different levels of impairment along the same continuum.
}

esional side of space (i.e., most commonly the left hemispace; Driver and Vuilleumier, 2001; Kerkhoff, 2001) extinction patients have difficulties in consciously perceiving a contralesional stimulus only when it is delivered simultaneously with an ipsilesional stimulation (Bender, 1952). This deficit specific to conditions of double simultaneous stimulation (DSS), together with a (at least almost) normal detection of contralesional stimuli presented in isolation, is the hallmark of extinction. Besides the widely recognized spatial nature of these two syndromes, extinction and neglect patients also exhibit deficits in the temporal processing of contralesional stimuli (Husain et al., 1997; Rorden et al., 1997). In particular, extinction patients exhibit an abnormally long attentional dwell-time (i.e., the period during which the perception of a first attended stimulus interferes with that of a subsequent one) in the contralesional visual hemifield, and this might contribute to the competitive bias in favor of ipsilesional stimuli in these patients (di Pellegrino et al., 1998). However, the temporal order of ipsi- and contralesional stimuli seems not to influence extinction rate, which remains maximal for simultaneous stimuli (di Pellegrino et al., 1997a; Rorden et al., 2009).

Neglect and extinction can affect all sensory modalities, either separately, across patients, or jointly, in the same individual (Brozzoli et al., 2006). Extinction was first reported in humans in the tactile 
modality by Oppenheim (1885; cited in Benton, 1956). The phenomenon has subsequently been described for visual (Poppelreuter, 1917; cited in Bender and Teuber, 1946; Critchley, 1953; Heilman et al., 1993) and auditory (Bender, 1952; Heilman et al., 1970; De Renzi et al., 1984; Jacquin-Courtois et al., 2010) stimuli as well. Cases of olfactory (Mesulam, 1981; Bellas et al., 1988a,b) and gustatory (Bender and Feldman, 1952) extinction have also been reported, although it is unclear whether they reflected true olfactory or gustatory extinction, or were rather due to somatosensory deficits (Kobal et al., 1989; Berlucchi et al., 2004).

The first observation of extinction in animals also dates back to 1885. In parallel to Oppenheim's discovery in humans, Loeb reported that dogs with damage to one hemisphere favored pieces of meat situated on the side of the lesion only upon simultaneous presentation of two pieces of meat (Benton, 1956). Since then, a few studies have sought to develop a model of neglect or extinction in non-human primates, using lesions of parietal, frontal, or superior temporal cortices to induce behaviors similar to those observed in patients. Authors have investigated the impact of these lesions on the perception of visual (Faugier-Grimaud et al., 1978; Lynch and McLaren, 1989; Watson et al., 1994; Heilman et al., 1995) or tactile (Ettlinger and Kalsbeck, 1962) stimuli, or both (but as separate modalities; Matelli et al., 1982; Rizzolatti et al., 1983). For example, Lynch and McLaren (1989) demonstrated visual extinction in five monkeys with unilateral parietal damage trained to detect single or double small visual targets presented at various eccentricities. Although still able to direct saccades toward a single contralesional stimulus, all five monkeys were unable to detect the same target when presented simultaneously with an ipsilesional one.

For about a century after its discovery, extinction was thought to occur only within sensory modalities, despite anecdotal reports of crossmodal extinction (Bender, 1952). As a unimodal phenomenon, it provided insights into the neural bases of unisensory competition (Kinsbourne, 1977; Duncan, 1996; Riddoch et al., 2009). However, when its multisensory dimension became clear, extinction could then be used as a model to study the multisensory neural representation of space. The present review focuses on this most recent development in extinction research and the considerable insight it brought into human spatial cognition, before proposing new developments in healthy individuals and non-human primates as the support for future research on extinction. The first sections provide a brief outline of the original demonstration of the existence of multisensory (or crossmodal) extinction, its role in the investigation of multisensory spatial representation, and an overview of the current theoretical accounts of extinction. In the second part of this paper, we propose two future developments for extinction research that we believe are key to deepen our understanding of the cerebral mechanisms of extinction and multisensory processing. The first one is the exploration of physiological extinction phenomena in healthy humans, their behavioral mechanisms and their neural underpinnings, thanks to functional neuroimaging and brain stimulation methods. The second one is the refinement of the existing non-human primate model of extinction by using the same behavioral approach as in humans to strengthen the validity for inter-species comparisons, also building on the unique opportunity provided by animal research of combining focal lesions techniques to functional neuroimaging methods.

\section{EXTINCTION AS A MULTISENSORY PHENOMENON}

Posner's seminal work on selective spatial attention in the 1980 s (Posner et al., 1982, 1984, 1987a,b) motivated the first attempts to systematically investigate whether stimuli presented in different sensory modalities could compete for common, supramodal attentional resources. The first evidence of multisensory competition for attentional resources was brought by Farah et al. (1989), who used a modified version of Posner's task (Posner et al., 1982). In this study, a lateralized auditory cue, either valid or invalid, preceded the presentation of a visual target either to the left or to the right of a central fixation point, and participants had to respond as soon as possible to the visual target by pressing the corresponding button. Patients with right parietal lesions were slower at detecting contralesional left visual targets when previously invalidly cued to the right side of space, suggesting a deficit in disengaging spatial attention from the wrongly cued ipsilesional location to subsequently relocate it to the contralesional visual target. Farah et al. (1989) interpreted these results as evidence for the existence of a supramodal system controlling the allocation of spatial attention. However, auditory cues did not interfere with the perception of visual stimuli to the point of hampering their detection. While these lengthened reaction times indeed reflected interferences between the processing of auditory cues and visual target stimuli, they remained much less spectacular than the outright omissions of stimulations observed in extinction patients during DSS.

Along the same line, Inhoff et al. (1992) investigated more explicitly the possible existence of crossmodal, visuotactile extinction in patients suffering from right parieto-frontal lesions and exhibiting unimodal visual and tactile extinction. To this end, they used the classical confrontation test, which consists in having the patient face the experimenter and apply stimuli either to the left or right side of space, or both simultaneously. Typically, the patient is asked to verbally report which side(s) the stimulation was applied to, and the experimenter compares performance on single stimulus detection with responses to DSS. As detailed previously, the hallmark of extinction is a (relatively) normal detection of single stimulations together with a deficit in detecting contralesional stimuli on DSS trials. In Inhoff et al.'s (1992) study, visual stimuli corresponded to wiggling either index finger or both at the patient's eye level, while tactile stimulations were applied by lightly touching the dorsum of the hand(s) while the patient had the eyes closed. The three patients tested showed pronounced extinction within the visual and tactile modalities, but no sign of visuotactile extinction, which led Inhoff et al. (1992) to conclude that processing of sensory inputs from both modalities is controlled by separate spatial attention systems, and that parieto-frontal brain structures may not contribute to the elaboration of a supramodal representation of space.

These findings were subsequently challenged by abundant neurophysiological data collected in the monkey that demonstrated the existence of multimodal neurons in areas commonly associated with spatial attention and extinction, such as parietal cortex (Duhamel et al., 1998) and basal ganglia (Graziano and Gross, 1993), as well as ventral premotor cortex (Rizzolatti et al., 1981; Graziano et al., 1994). These neurons respond to stimuli delivered in different sensory modalities (e.g., vision and touch) and have been suggested to constitute the substrate for crossmodal integration and multisensory representation of space. The most widely studied cells are visuotactile 
neurons, which have a visual receptive field in register with their tactile receptive field, that is, they respond to visual stimuli applied on or in the space immediately surrounding a specific body part, thus providing multiple multisensory maps of space centered on different body parts. Importantly, this implies that visuotactile interactions are strongest near the body, but fade as the visual stimulus moves away.

In light of these findings, Inhoff et al.'s (1992) failure to demonstrate the existence of visuotactile extinction might just be due to the fact that their visual stimulations were delivered too far from the hand, at patients' eye level, where they would interact much less, if at all, with the tactile stimuli. Consistent with this interpretation, di Pellegrino et al. (1997b) and Mattingley et al. (1997), independently and at about the same time, tested patients with right brain damage and suffering from unisensory extinction in the tactile or visual modality, or both. Both investigations were based on a confrontation test similar to that used in Inhoff et al.'s (1992) investigation, except that visual stimuli were applied near the hand. In both studies, patients exhibited strong visuotactile extinction, with a visual stimulus applied near the ipsilesional hand hampering the detection of a tactile stimulation of the contralesional hand. The reverse pattern of extinction (i.e., a right tactile stimulus extinguishing a left visual stimulus applied near the hand) was also present when investigated (Mattingley et al., 1997). Importantly, the patient tested in di Pellegrino et al.'s (1997b) study did not show visuotactile extinction when the ipsilesional visual stimulus was applied far from the hand, supporting the hypothesis that these multisensory interactions are supported by mechanisms similar to those described in the monkey (Rizzolatti et al., 1981; Graziano et al., 1997; Duhamel et al., 1998). However, the fact that crossmodal extinction was still present when the visual stimulus was applied at patients' eye level in Mattingley et al.'s (1997) study suggests that these interactions, although strongest in near space, are not an all-or-nothing phenomenon. This is consistent with subsequent observations that, although usually milder, crossmodal extinction can still occur with ipsilesional stimuli delivered far from the hand (Làdavas et al., 1998a; Farnè and Làdavas, 2000), suggesting that the transition from near to far space is gradual rather than an abrupt boundary at arm's length (Longo and Lourenco, 2006).

Since the demonstration of the existence of visuotactile extinction, many investigations have used extinction as a window on the mechanisms of normal multisensory processing in the human brain, showing for example that other sensory modalities can interact, such as touch and audition (Làdavas et al., 2001; for a similar demonstration using alloesthesia, see Ortigue et al., 2005). Most importantly, as we will detail in the next section, the study of neglect and crossmodal extinction has proven an invaluable tool for the exploration of human spatial cognition.

\section{MULTISENSORY EXTINCTION AND THE MODULAR REPRESENTATION OF SPACE}

Although our conscious experience of the space that surrounds us is that of a unitary entity, it is actually the product of the integration of multiple neural representations, subserved by distinct cerebral systems and involving the convergence of information perceived in different sensory modalities (Calvert et al., 2004; Spence and Driver, 2004). The first evidence of separate representations of distinct sectors of space in humans has been brought by the demonstration that neglect can be restricted to personal space (e.g., Bisiach et al., 1986) or to the space within (Halligan and Marshall, 1991; Berti and Frassinetti, 2000) or beyond (Vuilleumier et al., 1998) reaching distance. This distinction between the representations of near and far space had been previously shown in monkeys following focal lesions to the postarcuate cortex (area 6), which induced tactile and visual neglect for the contralateral space immediately surrounding the body, and to the frontal eye field (area 8), which gave rise to visual neglect for far space, with a tendency to deviate the eyes and the head toward the side of the lesion and to decrease spontaneous eye movements contralateral to the lesion, as typically observed in human neglect (Rizzolatti et al., 1983). Subsequently however, the study of patients with crossmodal extinction has provided a much more detailed picture of the modularity of the cerebral representation of space.

Indeed, the first report of spatially selective visuotactile extinction described above (di Pellegrino et al., 1997b) provided evidence that the space located within reaching distance, also often termed peripersonal space (for a detailed discussion of these concepts of spatial representation, see Cardinali et al., 2009), can be divided into two parts: a near sector, constituted of the space immediately surrounding body (about $5 \mathrm{~cm}$ ), where visuotactile integration is strongest; and a far sector (at approximately $35 \mathrm{~cm}$ from the body), where visuotactile interactions are weaker. In addition, these studies showed that the maps of near peripersonal space were centered on a specific body part, that is, in those cases, the hand. In particular, in one of their control conditions, di Pellegrino et al. (1997b) presented the visual stimulus at the same spatial location as in the regular confrontation test (i.e., next to where the patient's hand used to be), while the patient held his hand behind his back. In this condition, the patient detected flawlessly the left tactile stimulus when it was delivered concurrently with the ipsilesional visual stimulus, showing that the visuotactile interaction evidenced in the normal confrontation test occurred in hand-centered space, and not in a retinal or other more general egocentric (e.g., head- or trunk-centered) spatial frame of reference. This interpretation was further supported by the observation that, while a visual stimulus might prevent the detection of a contralesional tactile stimulus if it is applied near the ipsilesional hand in a patient with tactile extinction, it can on the contrary enhance the detection of the same contralesional tactile stimulus if it is instead applied near the contralesional hand during tactile DSS (Làdavas et al., 1998a). Again, this modulation of tactile perception by visual stimuli was dramatically diminished if the visual stimulus was applied far from the hand. Therefore, in the former case, the simultaneous activation of the visuotactile representations of the left and right hands by the tactile and visual stimulus, respectively, leads to the extinction of the weaker representation supported by the lesioned right hemisphere, that is, the one of the left hand. By contrast, in the latter case, when the visual stimulus is applied near the left hand, it enhances the activation of the weaker representation, thereby allowing the detection of a left tactile stimulus even when a concurrent tactile stimulus is delivered on the right hand.

Subsequently, similar paradigms have been used to reveal the existence of multisensory maps of near peripersonal space and their relation to other body parts. In particular, crossmodal extinction has been used to demonstrate interactions between vision 
and touch in a space centered on the face in humans (Làdavas et al., 1998b). In this study, extinction patients received unilateral and bilateral tactile stimuli on the cheeks, together with a visual stimulus applied either on the ipsilesional or on the contralesional side. Akin to what has been described for the hand, the visual stimulus extinguished the contralesional tactile stimulus when presented ipsilesionally, whereas it enhanced its detection when delivered near the contralesional cheek. Again, visual stimuli presented far from the face modulated touch perception to a much lesser extent. Further investigation strengthened the evidence of a modular organization of visuotactile near peripersonal space, by directly studying the effects of ipsilesional visual stimuli delivered to homologous or non-homologous body parts (i.e., the face or the hand) to that receiving the contralesional tactile stimulus (Farnè et al., 2005a). Visuotactile extinction observed in near peripersonal space was stronger when homologous body parts were stimulated, while it was weak and comparable in the homologous and nonhomologous condition in far peripersonal space. Near peripersonal space is thus not represented as a unitary entity, but rather consists of different modules, separately representing multisensory space, possibly centered on several body parts.

Finally, similar modulations of tactile extinction have been revealed through multisensory interactions between touch and audition (Làdavas et al., 2001; Farnè and Làdavas, 2002). Detection of contralesional single touches applied on the neck of tactile extinction patients was prevented by ipsilesional white noise stimuli delivered near the patients' head. When the auditory stimulus was delivered far from the head, audiotactile extinction was dramatically reduced. Interestingly, spatially selective interactions between audition and touch were strongest when the auditory stimuli came from the back, rather than from the front, of the patients' head. Additionally, the spatial specificity of the modulation of touch perception by audition was more pronounced for white noise bursts than pure tones. This suggests that different degrees of multisensory integration may occur depending on the complexity of the stimuli, and on the functional relevance of a given sensory modality for a particular sector of space (Farnè and Làdavas, 2002).

Together, these phenomena of crossmodal extinction and facilitation show that multisensory maps of near peripersonal space are elaborated through the integration of multisensory information, presumably at the neuronal level, via multimodal cells similar to those described in the monkey (Rizzolatti et al., 1981; Graziano and Gross, 1993; Graziano et al., 1994, 1997; Duhamel et al., 1998). As we have already mentioned, those neurons have been found in several cortical regions in the monkey that are commonly associated to spatial attention and extinction (Vallar et al., 1994; Karnath et al., 2003; Corbetta et al., 2005), including parietal (Duhamel et al., 1998) and ventral premotor (Rizzolatti et al., 1981; Graziano et al., 1994) cortices. This interpretation is supported by the fact that crossmodal extinction in humans and the activity of multimodal neurons in monkeys are modulated by similar parameters. Indeed, multimodal neurons, whether visuotactile (Rizzolatti et al., 1981; Graziano and Gross, 1993; Graziano et al., 1994; Duhamel et al., 1998) or audiotactile (Graziano et al., 1999), progressively cease to fire when the non-corporeal stimulus (i.e., visual or auditory) moves away from the relevant body part. Furthermore, similarly to audiotactile extinction described in patients (Farnè and Làdavas,
2002), the activity of monkey audiotactile neurons is also modulated by auditory complexity (Graziano et al., 1999). Finally, tool use induces plastic modifications of the multisensory representation of near space in both monkeys and humans. In monkeys, visuotactile neurons that normally fire only when visual stimuli are applied near the hand show an enlarged visual receptive field after tool use, which encompasses the hand and the tool (Iriki et al., 1996). In patients, visuotactile extinction increases strongly in the region of space that was previously beyond reach (i.e., far space) but made accessible by the use of the tool (for a similar effect in neglect patients, see Berti and Frassinetti, 2000; in healthy individuals, see Maravita et al., 2002; Holmes et al., 2004; for review, see Maravita and Iriki, 2004; Farnè et al., 2007). Additionally, this remapping occurs only after active and functional use of the tool in both species (Iriki et al., 1996; Maravita et al., 2002; Farnè et al., 2005b).

In sum, the body of evidence reviewed here and collected through the study of crossmodal extinction supports the hypothesis of a cerebral representation of multisensory space organized in a modular fashion, supported by several neural systems devoted to the representation of different sectors of space, in different coordinates, and probably for different behavioral purposes (Stein and Arigbede, 1972).

\section{THEORETICAL ACCOUNTS OF UNI- AND MULTISENSORY EXTINCTION}

As stated earlier, extinction and neglect are often considered manifestations of the same underlying deficit, representing different levels of impairment along a continuum (e.g., Heilman et al., 1993). As such, both disorders have usually been addressed jointly by several theories proposed to account for their behavioral manifestations. Whether they postulate a deficit in the representation of the contralesional hemispace (Bisiach et al., 1981) or in the ability to disengage attention from ipsilesional stimuli (Posner et al., 1984), a deviation (Jeannerod and Biguer, 1987) or a distortion (Bisiach et al., 1996) of spatial representation, most of these interpretations have been based on a dominant role of the right hemisphere in representing and orienting attention to both sides of space, while the left hemisphere would only be concerned with the right contralateral hemispace (Heilman et al., 1993, 1997). While this assumption might explain the spatial bias exhibited by neglect patients who fail to attend to or act toward stimuli in the contralesional hemispace, it is hard to reconcile with the competitive nature of extinction. Indeed, the hypothesis that the right lesion would leave intact only the attentional capacities of the left hemisphere leads to the prediction of an inability to detect single contralesional stimuli, rather than extinction arising from the competition between ipsi- and contralesional stimuli for attentional resources (for a detailed discussion of this point, see di Pellegrino and De Renzi, 1995). In fact, this view is hardly compatible with the competitive aspects of neglect itself, which has been shown to be reduced for example on a cancelation task when ipsilesional targets were progressively removed (Mark et al., 1988). This finding has been interpreted as evidence for a deficit in disengaging attention from ipsilesional stimuli, but might also be conceived of as suggesting competitive underlying mechanisms.

An alternative model addresses explicitly the competitive dimension of extinction (and neglect) by proposing that these phenomena result from a breakdown in the dynamic balance that normally exists 
in the reciprocal inhibition between homologous areas of the two hemispheres that orient spatial attention in opposing, contralateral directions (Kinsbourne, 1977, 1987). According to this proposal, a lesion lateralized to one hemisphere induces not only its hypoactivity, but also hyperactivity in the other hemisphere due to the release of inhibition by the affected one. This imbalance generates a general bias toward the ipsilesional side of space, which results in a biased competition between simultaneous stimuli (Duncan, 1996; Driver et al., 1997). The higher frequency of neglect and extinction following left than right brain damage would be explained by the fact that the rightward orienting bias generated by the left hemisphere is stronger than the opposing bias induced by the right hemisphere. This model provides a convincing explanation of why, in extinction, a contralesional stimulus is normally detected most of the time when delivered in isolation, but goes undetected when presented simultaneously with an ipsilesional stimulus (Bender, 1952). Because the deficit lies at the level of interhemispheric rivalry, in the former case, the lesioned hemisphere is still capable of processing (almost normally) the isolated contralesional stimulus. In the latter case, however, the concurrent activation of the intact hemisphere by the ipsilesional stimulus leads to enhanced inhibition of homologous areas on the side of the lesion, thereby preventing the conscious detection of the contralesional stimulus. Strong support to this model comes from brain stimulation studies using repetitive transcranial magnetic stimulation (rTMS) to temporarily decrease the activity of the intact hemisphere in brain-damaged patients suffering from tactile extinction (Oliveri et al., 1999) and neglect (Oliveri et al., 2001). In both cases, transient deactivation of the healthy hemisphere temporarily decreased the manifestations of extinction or neglect, in accordance with the predictions drawn from Kinsbourne's theory. Although the implications of this model have usually been discussed in the framework of unimodal extinction (e.g., di Pellegrino and De Renzi, 1995), it is applicable to the case of crossmodal extinction as well, whether the competition is considered to happen at the level of the orientation of some supramodal attentional system, or at a more physiological level where multisensory representations of near peripersonal space interact directly through reciprocal transcallosal connections.

The results of recent neuroimaging studies have led to a more complete picture of the pathophysiological mechanisms involved in neglect and extinction, and given further support to their competitive accounts. Corbetta et al. (2000) orienting of spatial visual attention toward a specific location involves a bilateral, dorsal frontoparietal network comprising the intra-parietal sulcus and superior parietal lobule, and the frontal eye field. Another, more ventral network, constituted of the temporoparietal and inferior parietal region and the caudal part of the inferior frontal gyrus, redirects attention toward novel stimuli by sending signals to the dorsal network. This ventral network is lateralized to the right hemisphere, and corresponds to the location of lesions usually associated with neglect. Further work in right brain-lesioned patients with neglect has shown that lesions in these patients not only directly affect the ventral attention network, but also indirectly modifies the activity within the structurally intact bilateral dorsal network (Corbetta et al., 2005). Indeed, the disruption of the normal interaction between the ventral and dorsal systems resulted in these patients in abnormally unbalanced activity in the left and right dorsal parietal cortices, with increased activations in the contralesional left hemisphere and comparatively reduced activity on the right side. Further, the hyperactivity of the left superior parietal lobe correlated with the patients' bias toward the ipsilesional side of space. These findings thus provide strong support to the competitive accounts of neglect and extinction, and suggest that dorsal parietal cortex is the site of the interhemispheric activity imbalance responsible for neglect and extinction patients' rightward spatial bias.

In the framework offered by these models, it is possible to make predictions about healthy individuals' behavior. Indeed, the interhemispheric rivalry hypothesis suggests that even in a healthy brain, competition occurs between simultaneous stimuli. In line with this hypothesis, previous work has shown, using positron emission tomography (PET), that simultaneous bilateral visual stimulation induces a reduction in response of the primary visual cortex when compared to the activation observed after contralateral single stimulation, thereby providing physiological support for the existence of interhemispheric rivalry occurring in the intact brain at early levels of processing (Fink et al., 2000). Thus, it should be possible to elicit extinction in healthy subjects performing a detection task with simple-feature stimuli, provided that the system is placed in challenging enough conditions. However, in contrast with patients' behavior, healthy individuals should exhibit no spatial bias in favor of one side or the other. Indeed, while Kinsbourne postulated that the higher occurrence of neglect and extinction following right brain damage was due to a stronger rightward attentional orienting bias generated by the left hemisphere, he suggested that, in the absence of lesion, "the right brain's opposing leftward bias [...] holds the left hemisphere's rightward bias in check" (Kinsbourne, 1987, p. 72). This predicts that no spatial bias should be observed in the physiological state. Further, according to Corbetta et al.'s (2005) model, imbalance between the activity of left and right dorsal parietal cortices only occurs following lesion to the ventral attention network, lateralized to the right hemisphere.

Therefore, the competition should be balanced between the two hemispheres in subjects in a normal state of vigilance (Matthias et al., 2009; see also Ciçek et al., 2007).

\section{CROSSMODAL EXTINCTION AS A PHYSIOLOGICAL PHENOMENON}

The idea that extinction observed in brain-damaged patients may be a pathological exacerbation of limits to the physiological capacities of the brain is not new. Bender himself, in his seminal monograph about disorders of perception (Bender, 1952), anecdotally reported what resembles extinction-like phenomena in normal subjects during tactile DSS on non-homologous body parts. Subjects frequently missed the first of a series of DSS when they were not aware of what body parts would be stimulated. This observation led Bender to assume that more consistent patterns of extinction-like behavior might be observed in healthy individuals with the appropriate testing paradigm and devices. More recent works have investigated competitive phenomena in healthy subjects in the visual (Duncan, 1984; Gorea and Sagi, 2002) and tactile (Meador et al., 2001; Marcel et al., 2004; Serino et al., 2008) domain, but the induction of extinctionlike patterns of performance required the use of complex experimental displays and demands (discrimination task, unbalanced stimuli, masking procedures, etc...) that are highly likely to rely on 
different processes than the mere detection of simultaneous simple-feature stimuli. Other groups were able to elicit extinction-like behaviors in healthy individuals, but induced to this purpose temporary and reversible "lesions" using either TMS or transcranial direct current stimulation (tDCS) to transiently impair sensory processing (Dambeck et al., 2006; Meister et al., 2006; Sparing et al., 2009; for review, see Oliveri and Caltagirone, 2006). Below, we will review recent data from our group and others showing that extinction-like phenomena can be elicited in healthy individuals without brain stimulation, using simple stimuli and tasks much more similar to the classical confrontation test, provided the appropriate experimental conditions are met, as Bender had predicted.

In accordance with the predictions drawn from the competitive accounts of extinction, our group sought to demonstrate the existence of tactile extinction in healthy individuals (Farnè et al., 2007). The hypothesis was that the somatosensory system is intrinsically limited when simultaneous stimuli compete to reach consciousness, so that extinction should manifest itself physiologically, provided the stimuli are challenging enough to perceive. To address this last point, the intensity of stimuli was first titrated independently on each side in order for the subject to detect $90 \%$ of left and right single stimulations, in order to avoid ceiling effects. During the experiment, neurologically healthy subjects performed a task as close as possible to the confrontation test, to investigate the same processes as those involved in pathological extinction. Specifically, participants were asked to localize tactile stimuli delivered to either or both index fingers by verbally responding "left," "right," "both," or "none" as fast as they could, while their hands were out of their sight. While subjects were able to report approximately $90 \%$ of single stimulations, as expected from the titration procedure, the rate of correct detection dropped to $78 \%$ for DSS. This score was lower than the joint probability of detecting both stimuli, confirming that this drop in performance was due to a genuine extinction phenomenon. Finally, in agreement with the prediction made by the hemispheric rivalry hypothesis, there was no spatial bias in the distribution of participants' errors, meaning that they missed equally often left and right stimuli when a stimulus was extinguished on DSS trials.

This study is the first demonstration of the existence of tactile extinction-like phenomena in healthy individuals, using a very simple task, as close as possible to the classical confrontation test used with patients, as well as simple-feature stimuli. This allows us to confidently believe that we are studying the very same processes that are at play in clinical extinction. We are currently investigating whether the same procedure applied to visual stimuli results in patterns of performance reminiscent of extinction patients' behavior. Preliminary data suggest that the observations made for touch hold for the visual modality. Indeed, participants miss stimuli on DSS trials while they perceive single stimulations almost perfectly and, again, do not seem to exhibit a spatial bias when failing to perceive one of two simultaneous stimuli.

Multisensory interactions in the intact brain have already been studied in many previous works. Of particular relevance to the question addressed here are studies about the Colavita effect, a phenomenon reflecting the fact that vision dominates over other sensory modalities in most experimental conditions. Colavita (1974) originally designed an experiment in which visual, auditory, or audiovisual stimuli were randomly presented, asking participants to report what they had perceived. Typically, on a certain number of bimodal trials, subjects missed the auditory stimulus and reported only the visual one, while they had no difficulties in reporting isolated stimuli. There has been lately a resurgence of interest in this phenomenon, and many studies have confirmed the existence of this effect under more controlled experimental conditions (for review, see Spence, 2009). While this effect is somewhat reminiscent of the manifestations of crossmodal extinction, it lacks a spatial dimension for the parallel with extinction and interhemispheric competition to be solid. Recently however, this effect of visual dominance has been extended to the perception of visuotactile stimuli (Hartcher-O'Brien et al., 2008), showing that people tend to miss the tactile stimulus much more often than the visual one on DSS trials with extinction. In this study, Hartcher-O'Brien et al. (2008) first tested their participants in a similar configuration to the audiovisual Colavita experiments done before, that is, with one hand (right) located centrally in front of them. The tactile and visual stimuli were presented from the same spatial location, at the tip of the subject's index finger. In a subsequent experiment, to establish a better correspondence with crossmodal extinction, these authors introduced a spatial dimension to their task, by placing each hand in its corresponding visual field and asking the subjects to fixate centrally. The visual and tactile stimuli could be delivered to either hand, resulting in bimodal trials that were either unilateral (i.e., both stimuli applied to the same hand) or bilateral (i.e., each hand received one of the two stimuli). The latter condition corresponds to the typical experimental set up usually used to test for visuotactile extinction. The results show that, even when both stimuli do not come from the same spatial location but are rather delivered on homologous body parts placed in different hemispaces, the Colavita visual dominance effect still holds, although in lesser proportions.

Following the approach we used to study physiological extinction in the tactile and visual modalities, we recently started to investigate visuotactile extinction-like effects in healthy participants. Tactile stimuli are applied to the tip of either index finger, while visual stimuli are delivered in the space immediately surrounding either hand. A similar intensity titration is used independently for visual and tactile stimuli, on the left and right side, in order for the subject to detect approximately $90 \%$ of the single stimuli of each of the four types ( 2 modalities $\times 2$ sides). Then, during the experiment itself, unilateral visual or tactile stimuli as well as bilateral visuotactile stimulations are randomly delivered while participants are asked to report whether they detected a stimulus, regardless of its modality, on the left, right, or both sides. Preliminary data indicate that visuotactile extinction-like effects can be elicited in healthy individuals using this protocol: participants fail to perceive one of the two stimuli on DSS trials, while perceiving almost perfectly either visual or tactile stimuli delivered in isolation. Here again, there seem not to be a spatial bias in the distribution of their errors.

In sum, it is possible, using well-controlled experimental conditions, to induce extinction-like phenomena in healthy individuals, and thus to establish a physiological model of uni- and multisensory competition. Such a model will allow testing hypotheses drawn from patients' behavior about physiological processes directly, as well as overcoming the potential confounds inherently associated with the study of clinical extinction when it comes to the investigation of the anatomo-functional bases of multisensory perception of competing stimuli. Indeed, although valuable contributions can be made by 
single case investigations using functional neuroimaging techniques (e.g., Rees et al., 2000; Sarri et al., 2006) as well as group studies using lesion overlapping methods (e.g., Karnath et al., 2003), the neuropsychological approach is limited due to the heterogeneity of lesion sites, sizes, and etiology, and by the plasticity following permanent brain damage. The use of neuroimaging and neurophysiological techniques in neurologically healthy individuals will allow investigating the neural underpinnings of physiological extinctionlike phenomena in different modalities without these limitations. After identification of these functional networks, brain stimulation methods such as TMS and tDCS will permit to examine the behavioral disturbances caused by the selective disruption of specific nodes of these networks in the intact brain. This approach will also allow a better comprehension of the anatomo-functional and pathophysiological bases of clinical extinction by comparing these findings to the specific aspects of pathological sensory competition phenomena, such as the ipsilesional spatial bias and the prevalence of extinction after right-hemisphere damage. In sum, thanks to this physiological model of sensory competition, it will be possible to test the validity of current theoretical accounts of extinction, and to better understand how multisensory processes are organized in the intact brain.

In addition to this human physiological model, a refined animal model of the cerebral bases of sensory competition would provide the unique opportunity to bridge the gap between investigations of multisensory processes at the level of single cells and of functional networks, and between studies of the functioning of intact and damaged systems. To this end, establishing a model of physiological extinction-like phenomena in monkeys following the same approach as used in neurologically healthy humans would constitute a very valuable tool in order to refine the framework provided by previous lesion studies in non-human primates.

\section{TOWARD A NON-HUMAN PRIMATE MODEL OF MULTISENSORY COMPETITION}

As suggested in the previous sections, the properties of multimodal neurons recorded in several brain structures including the parietal and premotor cortices in the monkey (Rizzolatti et al., 1981; Graziano and Gross, 1993; Graziano et al., 1994; Duhamel et al., 1998) suggest that these neurons likely constitute crucial nodes in crossmodal interactions and the building of multisensory representations of space. However, typical lesion-based approaches in the monkey have so far been only partially satisfactory.

Cortical ablation studies in monkeys have shown that neglect and extinction can occur following lesions of either the periarcuate frontal (Rizzolatti et al., 1983; Heilman et al., 1995) or the inferior parietal regions (Faugier-Grimaud et al., 1978; Lynch and McLaren, 1989). A first limitation of these studies lies in the fact that the choice of the areas to be lesioned was based on a fair, but putative homology of functional anatomy between humans and monkeys. A second limitation comes from the rapid spontaneous recovery that follows permanent lesions that were used in most studies, leaving only little testing time, sometimes only a few days, which is insufficient to explore the many different aspects of neglect and spatial attention uncovered by neuropsychological investigations in humans (Matelli et al., 1982; Heilman et al., 1995; but see also, e.g., Wardak et al., 2006). Further, this rapid recovery raises questions about the validity of the comparison with human neglect, as it might reflect a true difference in the cerebral organization of attention and space representation mechanisms between the two species, or be related to secondary factors such as lesion location and size, or extent of white matter damage. Third, although testing tasks were appropriate, they were not necessarily sensitive enough.

The emergence of fMRI in monkeys in the recent years provides an invaluable tool to bridge the gap between these findings in monkeys and the research conducted in humans. In particular, one promising perspective is to induce physiological extinctionlike phenomena in monkeys within and between sensory modalities using very similar procedures as the one used in humans. The unique advantage of monkey fMRI is that it can be combined with invasive techniques, in particular focal reversible lesions, and thus unequivocally demonstrate which neural network is actually crucial for managing the competition involved in tasks involving the perception of simultaneous sensory stimuli.

By applying the very same task in healthy humans and in trained monkeys to identify the networks involved in competitive sensory perception using fMRI, future work should allow establishing true homologies between humans and monkeys based on functional activations. Functionally identified target regions could then be temporarily and reversibly shut down (using muscimol, see e.g., Wardak et al., 2004, 2006) in order to investigate their causal role in producing behavioral extinction-like effects. In other words, the pitfalls of permanent ablations would be overcome, and the consistency of the results would be ascertained by multiple testing sessions. In addition, using fMRI, one could study the impact of a circumscribed lesion on the activity of the remaining competitive sensory perception network and therefore shed light on the pathophysiology of neglect/extinction. In addition, such a model would undoubtedly provide extremely valuable insight into more theoretical aspects of spatial awareness.

\section{CONCLUSION}

The study of pathological crossmodal extinction has allowed deepening significantly our knowledge about how multisensory processing contributes to human spatial cognition. It has revealed the modular organization of the neural representation of space, with the existence of multiple multisensory maps of different sectors of space likely centered on specific body parts, and allowed to suggest a parallel between behavioral findings in humans and multisensory processes described at the cellular level in non-human primates. It also laid the bases for the development of a model of multisensory competition in neurologically healthy humans. Now, this physiological model can in turn motivate the parallel investigation of competitive multisensory processes in humans and monkeys using complementary techniques in order to complete the framework of the cerebral organization of the perception of multisensory competitive stimuli and of selective spatial attention, in human and non-human primates, from the cellular level to that of functional networks.

\section{ACKNOWLEDGMENTS}

Alessandro Farnè is supported by INSERM, and Martine Meunier by CNRS. This work was funded by the ANR grants no. RPV08085CSA and ANR-O8-BLAN-0068-1, the AVENIR grant no. R05265CS, a scholar award from the James S. McDonnell Foundation, and the NEURODIS Foundation. 


\section{REFERENCES}

Becker, E., and Karnath, H. O. (2007). Incidence of visual extinction after left versus right hemisphere stroke. Stroke 38, 3172-3174.

Bellas, D. N., Novelly, R. A., Eskenazi, B., and Wasserstein, J. (1988a). The nature of unilateral neglect in the olfactory sensory system. Neuropsychologia 26, 45-52.

Bellas, D. N., Novelly, R. A., Eskenazi, B. and Wasserstein, J. (1988b). Unilateral displacement in the olfactory sense: a manifestation of the unilateral neglect syndrome. Cortex 24, 267-275.

Bender, M. B. (1952). Disorders in Perception: With Particular Reference to the Phenomena of Extinction and Displacement. Springfield, IL: Charles C. Thomas.

Bender, M. B., and Feldman, D. S. (1952). Extinction of taste sensation on double simultaneous stimulation. Neurology 2, 195-202.

Bender, M. B., and Teuber, H. L. (1946). Phenomena of fluctuation, extinction and completion in visual perception. Arch. Neurol. Psychiatry 55 627-658.

Benton,A. L. (1956). Jacques Loeb and the method of double stimulation. J. Hist. Med. Allied Sci. 11, 47-53.

Berlucchi, G., Moro, V., Guerrini, C., and Aglioti, S. (2004). Dissociation between taste and tactile extinction on the tongue after right brain damage. Neuropsychologia 42, 1007-1016.

Berti, A., and Frassinetti, F. (2000). When far becomes near: remapping of space by tool use. J. Cogn. Neurosci. 12, 415-420.

Bisiach, E., Capitani, E., Luzzatti, C., and Perani, D. (1981). Brain and conscious representation of outside reality. Neuropsychologia 19, 543-551.

Bisiach, E., Perani, D., Vallar, G., and Berti, A. (1986). Unilateral neglect: personal and extra-personal. Neuropsychologia 24, 759-767.

Bisiach, E., Pizzamiglio, L., Nico, D., and Antonucci, G. (1996). Beyond unilateral neglect. Brain 119, 851-857.

Brozzoli, C., Demattè, M. L., Pavani, F., Frassinetti, F., and Farnè, A. (2006). Neglect and extinction: within and between sensory modalities. Restor. Neurol. Neurosci. 24, 217-232.

Calvert, G., Spence, C., Stein, B. E., and MITCogNet. (2004). The Handbook of Multisensory Processes. Cambridge, MA: MIT press.

Cardinali, L., Brozzoli, C., and Farnè, A. (2009). Peripersonal space and body schema: two labels for the same concept? Brain Topogr. 21, 252-260.

Ciçek, M., Gitelman, D., Hurley, R. S., Nobre, A., and Mesulam, M. (2007). Anatomical physiology of spatial extinction. Cereb. Cortex 17, 2892-2898.
Cocchini, G., Cubelli, R., Della Sala, S., and Beschin, N. (1999). Neglect without extinction. Cortex 35, 285-313.

Colavita, F. B. (1974). Human sensory dominance. Percept. Psychophys. 16, 409-412.

Corbetta, M., Kincade, J. M., Ollinger, J. M., McAvoy, M. P., and Shulman, G. L. (2000). Voluntary orienting is dissociated from target detection in human posterior parietal cortex. Nat. Neurosci. 3, 292-297.

Corbetta, M., Kincade, M., Lewis, C., Snyder, A., and Sapir, A. (2005). Neural basis and recovery of spatial attention deficits in spatial neglect. Nat. Neurosci. 8, 1603-1610.

Critchley, M. (1953). The Parietal Lobes. New York: Hafner.

Dambeck, N., Sparing, R., Meister, I. G., Wienemann, M., Weidemann, J., Topper, R., and Boroojerdi, B. (2006). Interhemispheric imbalance during visuospatial attention investigated by unilateral and bilateral TMS over human parietal cortices. Brain Res. 1072, 194-199.

De Renzi, E., Gentilini, M., and Pattacini, F. (1984).Auditory extinction following hemisphere damage. Neuropsychologia 22, 733-744.

di Pellegrino, G., Basso, G., and Frassinetti, F. (1997a). Spatial extinction on double asynchronous stimulation. Neuropsychologia 35, 1215-1223.

di Pellegrino, G., Làdavas, E., and Farnè, A. (1997b). Seeing where your hands are. Nature 388, 730.

di Pellegrino, G., Basso, G., and Frassinetti, F. (1998). Visual extinction as a spatiotemporal disorder of selective attention. Neuroreport 9, 835-839.

di Pellegrino, G., and De Renzi, E. (1995). An experimental investigation on the nature of extinction. Neuropsychologia 33, 153-170.

Driver, J., Mattingley, J. B., Rorden, C., and Davis, G. (1997). "Extinction as a paradigm measure of attentional bias and restricted capacity following brain injury," in Parietal Lobe Contributions to the Orientation in 3D Space, eds P. Thier and H. O. Karnath (Heidelberg: Springer), 401-430.

Driver, J., and Vuilleumier, P. (2001) Perceptual awareness and its loss in unilateral neglect and extinction. Cognition 79, 39-88.

Duhamel, J. R., Colby, C. L., and Goldberg, M.E. (1998). Ventral intraparietal area of the macaque: congruent visual and somatic response properties. J. Neurophysiol. 79, 126-136.

Duncan, J. (1984). Selective attention and the organization of visual information. J. Exp. Psychol. Gen. 113, 501-517.

Duncan, J. (1996). "Cooperating brain systems in selective perception and action," in Attention and Performance
XVI: Information Integration in Perception and Communication, eds T. Inui and J.L. McClelland (Cambridge, MA: MIT Press), 549-578.

Ettlinger, G., and Kalsbeck, J. E. (1962). Changes in tactile discrimination and in visual reaching after successive and simultaneous bilateral posterior parietal ablations in the monkey. J. Neurol. Neurosurg. Psychiatr. 25 256-268.

Farah, M. J., Wong, A. B., Monheit, M. A., and Morrow, L. A. (1989). Parietal lobe mechanisms of spatial attention: modality-specific or supramodal? Neuropsychologia 27, 461-470.

Farnè, A., Brozzoli, C., Làdavas, E., and Ro, T. (2007). "Investigating multisensory spatial cognition through the phenomenon of extinction," in Attention and Performance XXII, eds P. Hagard, Y. Rossetti, and M. Kawato (Oxford: Oxford University Press), 183-206.

Farnè, A., Demattè, M. L., and Làdavas, E. (2005a). Neuropsychological evidence of modular organization of the near peripersonal space. Neurology 65, 1754-1758.

Farnè, A., Bonifazi, S., and Làdavas, E. (2005b). The role played by tool-use and tool-length on the plastic elongation of peri-hand space: a single case study. Cogn. Neuropsychol. 22, 408-418.

Farnè,A., and Làdavas, E. (2000). Dynamic size-change of hand peripersonal space following tool use. Neuroreport $11,1645-1649$.

Farnè,A., and Làdavas, E. (2002). Auditory peripersonal space in humans. J. Cogn. Neurosci. 14, 1030-1043.

Faugier-Grimaud, S., Frenois, C., and Stein, D. G. (1978). Effects of posterior parietal lesions on visually guided behavior in monkeys Neuropsychologia 16, 151-168.

Fink, G. R., Driver, J., Rorden, C. Baldeweg, T., and Dolan, R. J. (2000). Neural consequences of competing stimuli in both visual hemifields: a physiological basis for visual extinction. Ann. Neurol. 47, 440-446.

Geeraerts, S., Lafosse, C., Vandenbussche, E., and Verfaillie, K. (2005). A psychophysical study of visual extinction: ipsilesional distractor interference with contralesional orientation thresholds in visual hemineglect patients. Neuropsychologia 43, 530-541.

Gorea, A., and Sagi, D. (2002). Natura extinction: a criterion shift phenomenon. Vis. Cogn. 9, 913-936.

Graziano, M.S., and Gross, C. G. (1993).A bimodal map of space: somatosensory receptive fields in the macaque putamen with corresponding visual receptive fields. Exp. Brain Res. 97, 96-109. Graziano, M.S., Hu, X. T., and Gross, C. G. (1997).Visuospatial properties of ven- tral premotor cortex. J. Neurophysiol. 77, 2268-2292.

Graziano, M. S., Reiss, L. A., and Gross, C. G. (1999). A neuronal representation of the location of nearby sounds. Nature 397, 428-430.

Graziano, M. S., Yap, G. S., and Gross, C. G. (1994). Coding of visual space by premotor neurons. Science 266, 1054-1057.

Halligan, P.W., and Marshall, J. C. (1991). Left neglect for near but not far space in man. Nature 350, 498-500.

Hartcher-O'Brien, J., Gallace, A., Krings, B., Koppen, C., and Spence, C. (2008) When vision 'extinguishes' touch in neurologically-normal people: extending the Colavita visual dominance effect. Exp. Brain Res. 186, 643-658.

Heilman, K. M., Pandya, D. N., and Geschwind, N. (1970). Trimodal inattention following parietal lobe ablations. Trans. Am. Neurol. Assoc. 95, 259-261.

Heilman, K. M., Valenstein, E., Day, A. and Watson, R. (1995). Frontal lobe neglect in monkeys. Neurology 45, 1205-1210.

Heilman, K. M., Watson, R. T., and Valenstein, E. (1993). "Neglect and related disorders," in Clinical Neuropsychology, 3rd Edn., eds K. M. Heilman and E. Valenstein (New York: Oxford University Press) 279-336.

Heilman K. M., Watson, R. T., and Valenstein,E. (1997). "Neglect: clinical and anatomic aspects," in Behavioral Neurology and Neuropsychology, eds T. E. Fineberg and M. J. Farah (New York: McGraw-Hill), 314.

Holmes, N. P., Calvert, G. A., and Spence, C. (2004). Extending or projecting peripersonal space with tools? Multisensory interactions highlight only the distal and proximal ends of tools. Neurosci. Lett. 372, 62-67.

Husain, M., Shapiro, K., Martin, J., and Kennard, C. (1997). Abnormal temporal dynamics of visual attention in spatial neglect patients. Nature 385, 154-156.

Inhoff, A. W., Rafal, R. D., and Posner, M. J. (1992). Bimodal extinction without cross-modal extinction. J. Neurol. Neurosurg. Psychiatr. 55, 36-39.

Iriki, A., Tanaka, M., and Iwamura, Y. (1996). Coding of modified body schema during tool use by macaque postcentral neurones. Neuroreport 7, 2325-2330.

Jacquin-Courtois, S., Rode, G., Pavani, F., O'Shea, J., Giard, M. H., Boisson, D., and Rossetti, Y. (2010). Effect of prism adaptation on left dichotic listening deficit in neglect patients: glasses to hear better? Brain 133, 895-908.

Jeannerod, M., and Biguer, B. (1987). “The directional coding of reaching move- 
ments. A visuomotor conception of spatial neglect," in Neurophysiological and Neuropsychological Aspects of Spatial Neglect, Vol. 45 (Amsterdam: North-Holland), 87-113.

Karnath, H. O., Himmelbach, M., and Küker, W. (2003). The cortical substrate of visual extinction. Neuroreport 14, 437-442.

Kerkhoff, G. (2001). Spatial hemineglect in humans. Prog. Neurobiol. 63, 1-27.

Kinsbourne, M. (1977). Hemi-neglect and hemisphere rivalry. Adv. Neurol. 18, 41-49.

Kinsbourne, M. (1987). "Mechanisms of unilateral neglect," in Neurophysiological and Neuropsychological Aspects of Spatial Neglect, eds M. Jeannerod (Amsterdam: Elsevier Science Publishers B.V.NorthHolland), 69-86.

Kobal, G., Van Toller, S., and Hummel, T. (1989). Is there directional smelling? Experientia 45, 130-132.

Làdavas, E., di Pellegrino, G., Farnè, A., and Zeloni, G. (1998a). Neuropsychological evidence of an integrated visuotactile representation of peripersonal space in humans. J. Cogn. Neurosci. 10, 581-589.

Làdavas, E., Zeloni, G., and Farnè, A. (1998b). Visual peripersonal space centred on the face in humans. Brain 121(Pt 12), 2317-2326.

Làdavas, E., and Farnè, A. (2004). Visuotactile representation of near-the-body space. J. Physiol. Paris 98, 161-170.

Làdavas, E., Pavani, F., and Farnè, A. (2001). Auditory peripersonal space in humans: a case of auditory-tactile extinction. Neurocase 7, 97-103.

Longo, M. R., and Lourenco, S. F. (2006). On the nature of near space: effects of tool use and the transition to far space. Neuropsychologia 44, 977-981.

Lynch, J. C., and McLaren, J. W. (1989). Deficits of visual attention and saccadic eye movements after lesions of parietooccipital cortex in monkeys. J. Neurophysiol. 61, 74-90.

Maravita, A., and Iriki, A. (2004). Tools for the body (schema). Trends Cogn. Sci. 8, 79-86.

Maravita, A., Spence, C., Kennett, S., and Driver, J. (2002). Tool-use changes multimodal spatial interactions between vision and touch in normal humans. Cognition 83, B25-B34.

Marcel,A., Postma, P., Gillmeister, H., Cox, S., Rorden, C., Nimmo-Smith, I., and Mackintosh, B. (2004). Migration and fusion of tactile sensation - premorbid susceptibility to allochiria, neglect and extinction? Neuropsychologia 42, 1749-1767.

Mark, V.W., Kooistra, C.A., and Heilman, K. M. (1988). Hemispatial neglect affected by non-neglected stimuli. Neurology 38, 1207-1211.

Matelli, M., Pavesi, G., and Rizzolatti, G. (1982). Unilateral lesions of the periarcuate cortex in the monkey. Boll. Soc. Ital. Biol. Sper. 58, 66-70.

Matthias, E., Bublak, P., Costa, A., Müller, H. J., Schneider, W. X., and Finke, K. (2009).Attentional and sensory effects of lowered levels of intrinsic alertness. Neuropsychologia 47, 3255-3264.

Mattingley, J. B., Driver, J., Beschin, N., and Robertson, I. H. (1997). Attentional competition between modalities: extinction between touch and vision after right hemisphere damage. Neuropsychologia 35, 867-880.

Meador, K. J., Ray, P. G., Day, L. J., and Loring, D. W. (2001). Relationship of extinction to perceptual thresholds for single stimuli. Neurology 56, 1044-1047.

Meister, I. G., Wienemann, M., Buelte, D., Grünewald, C., Sparing, R., Dambeck, N., and Boroojerdi, B. (2006). Hemiextinction induced by transcranial magnetic stimulation over the right temporo-parietal junction. Neuroscience 142, 119-123.

Mesulam, M. M. (1981). A cortical network for directed attention and unilateral neglect. Ann. Neurol. 10, 309-325.

Oliveri, M., Bisiach, E., Brighina, F., Piazza, A., La Bua, V., Buffa, D., and Fierro, B. (2001). rTMS of the unaffected hemisphere transiently reduces contralesional visuospatial hemineglect. Neurology 57, 1338-1340.

Oliveri, M., and Caltagirone, C. (2006). Suppression of extinction with TMS in humans: from healthy controls to patients. Behav. Neurol. 17, 163-167.

Oliveri, M., Rossini, P. M., Traversa, R. Cicinelli, P., Filippi, M. M., Pasqualetti, P., Tomaiuolo, F., and Caltagirone, C. (1999). Left frontal transcranial magnetic stimulation reduces contralesional extinction in patients with unilateral right brain damage. Brain 122(Pt 9), 1731-1739.

Ortigue, S., Jabaudon, D., Landis, T., Michel, C. M., Maravita, A., and Blanke, O. (2005). Preattentive interference between touch and audition: a case study on multisensory alloesthesia. Neuroreport 16, 865-868.

Posner, M. I., Cohen, Y., and Rafal, R. D. (1982). Neural systems control of spatial orienting. Philos. Trans. R. Soc. Lond. B Biol. Sci. 298, 187-198.
Posner, M. I., Inhoff, A. W., Friedrich, F. J., and Cohen, A. (1987a). Isolating attentional systems: a cognitive-anatomical analysis. Psychobiology 15, 107-121.

Posner, M. I., Walker, J. A., Fried, F. A. and Rafal, R. D. (1987b). How do the parietal lobes direct covert attention? Neuropsychologia 25, 135-145.

Posner, M. I., Walker, J. A., Friedrich, F. J., and Rafal, R. D. (1984). Effects of parietal injury on covert orienting of attention. J. Neurosci. 4, 1863-1874.

Rees, G., Wojciulik, E., Clarke, K., Husain, M., Frith, C., and Driver, J. (2000). Unconscious activation of visual cortex in the damaged right hemisphere of a parietal patient with extinction. Brain 123(Pt 8), 1624-1633.

Riddoch, M. J., Rappaport, S. J., and Humphreys, G. W. (2009). Extinction: a window into attentional competition. Prog. Brain Res. 176, 149-159.

Rizzolatti, G., Matelli, M., and Pavesi, G. (1983). Deficits in attention and movement following the removal of postarcuate (area 6) and prearcuate (area 8) cortex in macaque monkeys. Brain 106(Pt 3), 655-673.

Rizzolatti, G., Scandolara, C., Matelli, M. and Gentilucci, M. (1981). Afferent properties of periarcuate neurons in macaque monkeys. II.Visual responses. Behav. Brain Res. 2, 147-163.

Rorden, C., Jelsone, L., Simon-Dack, S. Baylis, L. L., and Baylis, G. C. (2009). Visual extinction: the effect of temporal and spatial bias. Neuropsychologia 47, 321-329.

Rorden, C., Mattingley, J. B., Karnath,H.O. and Driver, J. (1997). Visual extinction and prior entry: impaired perception of temporal order with intact motion perception after unilateral parietal damage. Neuropsychologia 35, 421-433.

Sarri, M., Blankenburg, F., and Driver, J. (2006). Neural correlates of crossmodal visual-tactile extinction and of tactile awareness revealed by fMRI in a right-hemisphere stroke patient. Neuropsychologia 44, 2398-2410.

Serino, A., Pizzoferrato, F., and Làdavas, E (2008).Viewing a face (especially one's own face) being touched enhances tactile perception on the face. Psychol. Sci. 19, 434-438.

Sparing, R., Thimm, M., Hesse, M. D. Kust, J., Karbe, H., and Fink, G. R. (2009). Bidirectional alterations of interhemispheric parietal balance by non-invasive cortical stimulation. Brain 132, 3011-3020.

Spence, C. (2009). Explaining the Colavita visual dominance effect. Prog. Brain Res. 176, 245-258.
Spence, C., and Driver, J. (2004) Crossmodal Space and Crossmodal Attention. Oxford: Oxford University Press.

Stein, B. E., and Arigbede, M. O. (1972). Unimodal and multimodal response properties of neurons in the cat's superior colliculus. Exp. Neurol. 36, 179-196.

Vallar, G., Rusconi, M. L., Bignamini, L., Geminiani, G., and Perani, D. (1994). Anatomical correlates of visual and tactile extinction in humans: a clinical CT scan study. J. Neurol. Neurosurg. Psychiatry 57, 464-470.

Vuilleumier, P., Valenza, N., Mayer, E., Reverdin, A., and Landis, T. (1998). Near and far visual space in unilateral neglect. Ann. Neurol. 43, 406-410.

Wardak, C., Ibos, G., Duhamel, J. R., and Olivier, E. (2006). Contribution of the monkey frontal eye field to covert visual attention. J. Neurosci. 26, 4228-4235.

Wardak, C., Olivier, E., and Duhamel, J. R. (2004). A deficit in covert attention after parietal cortex inactivation in the monkey. Neuron 42, 501-508.

Watson, R. T., Valenstein, E., Day, A., and Heilman, K. M. (1994). Posterior neocortical systems subserving awareness and neglect. Neglect associated with superior temporal sulcus but not area 7 lesions. Arch. Neurol. 51, 1014-1021.

Conflict of Interest Statement: The authors declare that the research was conducted in the absence of any commercial or financial relationships that could be construed as a potential conflict of interest.

Received: 21 January 2011; accepted: 26 April 2011; published online: 06 May 2011.

Citation: Jacobs S, Brozzoli C, HadjBouziane F, Meunier Mand Farnè A (2011) Studying multisensory processing and its role in the representation of space through pathological and physiological crossmodal extinction. Front. Psychology 2:89. doi: 10.3389/fpsyg.2011.00089

This article was submitted to Frontiers in Perception Science, a specialty of Frontiers in Psychology.

Copyright (c) 2011 Jacobs, Brozzoli, HadjBouziane, Meunier and Farnè. This is an open-access article subject to a nonexclusive license between the authors and Frontiers Media SA, which permits use, distribution and reproduction in other forums, provided the original authors and source are credited and other Frontiers conditions are complied with. 